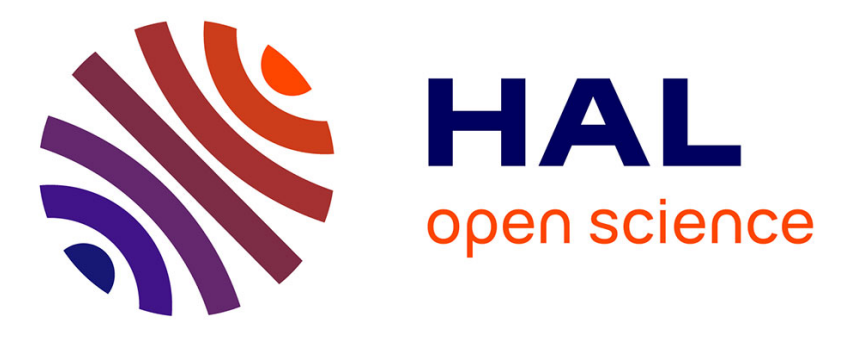

\title{
In situ synchrotron X-ray diffraction study of strain induced crystallisation of natural rubber during fatigue tests
}

\author{
Stéphanie Beurrot, Bertrand Huneau, Erwan Verron, Pierre Rublon, \\ Dominique Thiaudière, Cristian Mocuta, A. Zozulya
}

\section{To cite this version:}

Stéphanie Beurrot, Bertrand Huneau, Erwan Verron, Pierre Rublon, Dominique Thiaudière, et al.. In situ synchrotron X-ray diffraction study of strain induced crystallisation of natural rubber during fatigue tests. Plastics, Rubber and Composites, 2012, 41 (7), pp.290-295. 10.1179/1743289812Y.0000000028. hal-01006959

\section{HAL Id: hal-01006959 \\ https://hal.science/hal-01006959}

Submitted on 27 Sep 2017

HAL is a multi-disciplinary open access archive for the deposit and dissemination of scientific research documents, whether they are published or not. The documents may come from teaching and research institutions in France or abroad, or from public or private research centers.
L'archive ouverte pluridisciplinaire HAL, est destinée au dépôt et à la diffusion de documents scientifiques de niveau recherche, publiés ou non, émanant des établissements d'enseignement et de recherche français ou étrangers, des laboratoires publics ou privés. 


\title{
In situ synchrotron X-ray diffraction study of strain induced crystallisation of natural rubber during fatigue tests
}

\author{
S. Beurrot-Borgarino* ${ }^{1}$, B. Huneau ${ }^{1}$, E. Verron ${ }^{1}$, P. Rublon ${ }^{1}$, D. Thiaudière ${ }^{2}$, \\ C. Mocuta ${ }^{2}$ and A. Zozulya ${ }^{2}$
}

A homemade stretching machine has been developed to perform fatigue tests on natural rubber in the synchrotron facility Soleil. Strain induced crystallisation is investigated by wide angle X-ray diffraction during in situ fatigue tests of different minimum and maximum strain levels. The index of crystallinity $\chi$ decreases with the number of cycles when the minimum strain level reached during the fatigue test is lower than the critical stretch ratio for melting $\lambda_{\mathrm{M}}$. On the contrary, when the stretch ratio is maintained higher than the critical stretch ratios for melting $\lambda_{M}$ and crystallisation $\lambda_{\mathrm{c}}, \chi$ increases with the number of cycles. The size of the crystallites has the same evolution during fatigue testing as $\chi$. On the contrary, the misorientation of the crystallites does not depend on the minimum strain level reached and decreases with the number of cycles for all the fatigue tests.

Keywords: Natural rubber, Strain induced crystallisation, WAXD, Fatigue

\section{Introduction}

Natural rubber (NR), cis-1,4-polyisoprene, has remarkable fatigue properties ${ }^{1,2}$ that are generally explained by strain induced crystallisation (SIC). The SIC is commonly investigated by wide angle X-ray diffraction (WAXD). However, studies on the evolution of SIC during fatigue testing are very rare mainly because the typical frequencies of fatigue tests $(1 \mathrm{~Hz}$ or more) are not compatible with the long time acquisition required by X-ray diffraction measurements (from a few seconds to an hour). Kawai ${ }^{3}$ succeeded in measuring SIC during fatigue using a stroboscopic technique to accumulate the weak intensity of the diffracted beam over several hundreds of cycles. However, this technique requires to average the crystallinity over a large number of cycles. Moreover, this work was limited to a large $R$ ratio $\left(R=\lambda_{\min } / \lambda_{\max }=3 \cdot 5 / 4 \cdot 5\right)$ for which crystallites never melt because $\lambda_{\min }$ always remains greater than the critical stretch ratio for melting. Furthermore, Rouvière et al. recently performed interrupted fatigue tests and WAXD measurements on stretched fatigued samples to obtain the evolution of the crystallinity along fatigue life for different values of $R .{ }^{4}$ However, this method does not allow to separate SIC due to fatigue from SIC due to constant elongation during the $45 \mathrm{~min}$ acquisition of the X-ray diffractogram.

\footnotetext{
${ }^{1}$ LUNAM Université, Ecole Centrale de Nantes, GeM, UMR CNRS 6183 BP 92101, Nantes cedex 3 44321, France

${ }^{2}$ Synchrotron Soleil, Gif Sur Yvette 91192, France

*Corresponding author, email stephanie.beurrot@ec-nantes.fr
}

In the present study, we use synchrotron radiation to reduce the exposure time and to perform in situ fatigue tests for different $R$ ratios. A versatile testing machine allowing uniaxial and biaxial loading conditions as well as large strain was especially designed and built for that purpose.

\section{Experimental}

\section{Material and sample}

The material used in this study is a carbon black filled NR crosslinked with 1.2 per hundred of rubber (phr) of sulphur and N-cyclohexyl-2-benzothiazyl-sulfenamide (CBS) accelerator. It also contains $\mathrm{ZnO}$ (5 phr) and stearic acid (2 phr) and is filled with $50 \mathrm{phr}$ of N330 carbon black. The samples are classical flat dumbbell specimen with a $10 \mathrm{~mm}$ gauge length and a $2 \times 4 \mathrm{~mm}$ section.

\section{Synchrotron}

The synchrotron measurements have been carried out at the beamline in the French national synchrotron facility Soleil. The wavelength used is $1.319 \AA$, and the beam size is $0.3 \mathrm{~mm}$ in diameter at half maximum. The two-dimensional WAXD patterns are recorded by an MAR 345 CCD X-ray detector. In order to make an accurate correction of air scattering, a PIN diode beam stop was used.

\section{Fatigue testing machine}

Fatigue tests have been conducted with a homemade stretching machine shown in Fig. 1. It is composed of four electrical actuators, but only two opposite ones were used in this study. Their movements are synchronised in order to keep the centre of the specimen fixed 


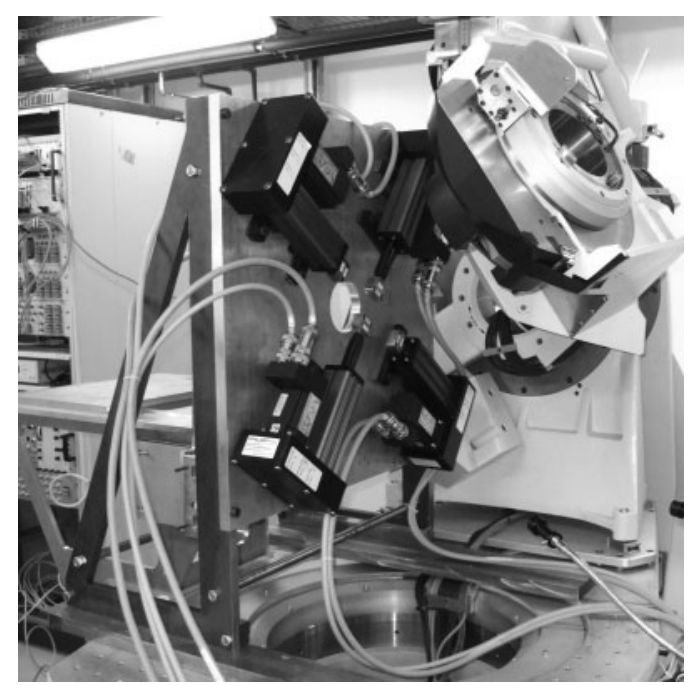

1 Uniaxial and biaxial stretching machine in DiffAbs, Soleil, France

during the fatigue tests. Their loading capacity is $\pm 500 \mathrm{~N}$, and their stroke is $75 \mathrm{~mm}$ each.

\section{Procedure}

All the experiments are conducted by prescribing constant displacements of the clamps at each cycle. Just before fatigue testing, specimens are first cycled at a higher deformation (during 55 cycles) in order to lower the remaining elongation of the sample due to Mullins effect and viscoelasticity during the fatigue tests.

The minimum exposure time of the CCD detector to record a workable scattering pattern is $1 \mathrm{~s}$, which is about the duration of a fatigue cycle. It is then not possible to record diffraction patterns during fatigue tests while the actuators are in motion. Hence, to measure the evolution of crystallisation during fatigue testing, it has been decided to pause the test at maximum deformation of the sample every 250 cycles to record a complete diffraction pattern. The fatigue machine is triggered by the monitoring system of the X-ray beam, and the duration of the pause is $<1.5 \mathrm{~s}$. The first scattering pattern is recorded during the first cycle of a test.

Four different fatigue tests have been performed; Table 1 shows for each the minimum and maximum displacements of the grips $l_{\min }$ and $l_{\max }$ and the loading frequency $f$.

\section{Scattering pattern analysis}

An air scattering pattern (without sample) was first collected and has been used to correct the patterns. Moreover, the change in thickness of the sample under extension and the change of intensity of the incident photons have also been considered. All these corrections are performed by following the well established method of Ran et $a l^{5}$ Both the determination of the pattern

Table 1 Fatigue loading conditions

\begin{tabular}{llll}
\hline Test no. & $\boldsymbol{I}_{\mathbf{m i n}} / \mathbf{m m}$ & $\boldsymbol{I}_{\max } / \mathbf{m m}$ & $\boldsymbol{f} / \mathrm{Hz}$ \\
\hline 1 & 0 & 20 & $2 \cdot 5$ \\
2 & 4 & $33 \cdot 2$ & $0 \cdot 8$ \\
3 & $9 \cdot 3$ & $33 \cdot 2$ & 1 \\
4 & 25 & 45 & $1 \cdot 5$ \\
\hline
\end{tabular}

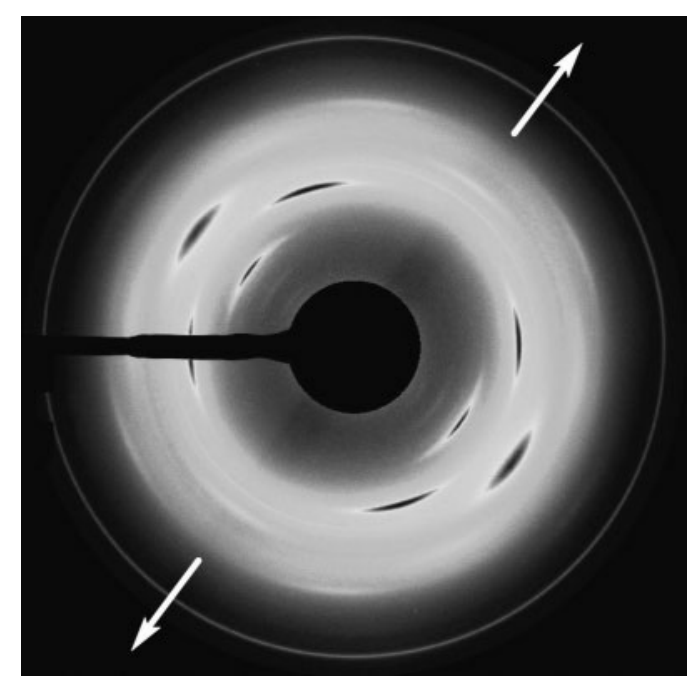

2 Example of diffraction pattern. Dark arcs stem from crystalline phase, whereas large light ring is reflexion of amorphous phase. White arrows show tensile direction

centre and the calibration of the diffraction angles were achieved by considering the first diffraction ring of $\mathrm{ZnO}$ [(100) plane, $a=3 \cdot 25 \AA$ ] ${ }^{6}$ Here, small angle scattering was not investigated; the range of diffraction angles is $2 \theta \in\left[8^{\circ}, 26 \cdot 7^{\circ}\right.$. An example of the diffraction pattern is shown in Fig. 2.

The intensity of photons diffracted by the isotropic phases in the material $I_{\text {isotropic }}(2 \theta)$ is extracted from the diffraction patterns by considering the minimum intensity along the azimuthal angle $\beta$ for each Bragg angle $2 \theta$. Then, the intensity of photons diffracted by the anisotropic material $I_{\text {anisotropic }}(2 \theta, \beta)$ is calculated as the difference between the total intensity of photons diffracted $I_{\text {total }}(2 \theta, \beta)$ and $I_{\text {isotropic }}(2 \theta)$. The spectra extracted from $I_{\text {anisotropic }}(2 \theta, \beta)$ and $I_{\text {isotropic }}(2 \theta)$ are classically fitted by a series of Pearson functions. ${ }^{7-10}$ Figure $3 a$ and $b$ presents examples of fitting and deconvolution of a $\left(2 \theta, I_{\text {isotropic }}\right)$ spectrum and a $\left(2 \theta, I_{\text {anisotropic }}\right)$ spectrum respectively.

The index of crystallinity $\chi$ is calculated from the simplified Mitchell formula ${ }^{11}$

$$
\chi=\frac{\Im_{\text {cryst }}}{\Im_{\text {cryst }}+\Im_{\text {amorphous }}}
$$

where $\Im_{\text {cryst }}$ is the integrated intensity of the (120) and (200) Bragg reflections of NR, and $\Im_{\text {amorphous }}$ is the integrated intensity of the amorphous halo, considered equal to the integrated intensity $I_{\text {isotropic }}$.

The crystallite size is deduced from the Scherrer formula $^{12}$

$$
l_{\mathrm{hkl}}=\frac{K \lambda}{\mathrm{FWMH}_{2 \theta} \cos \theta}
$$

where $l_{\mathrm{hkl}}$ is the crystallite size in the direction normal to the $h k l$ diffraction plane, $K$ is a scalar that depends on the shape of crystallites (here we adopt 0.78 as Trabelsi et $\left.a l^{8}\right), \lambda$ is the radiation wavelength, $\beta$ is the Bragg angle and $\mathrm{FWHM}_{2 \theta}$ is the full width at half maximum of the peak $h k l$ in $2 \theta$. Finally, the crystallite misorientation is simply given by half the full width at half maximum $\left(\mathrm{FWHM}_{\beta}\right)$ of the peaks, measured on the azimuthal profiles of reflection. 

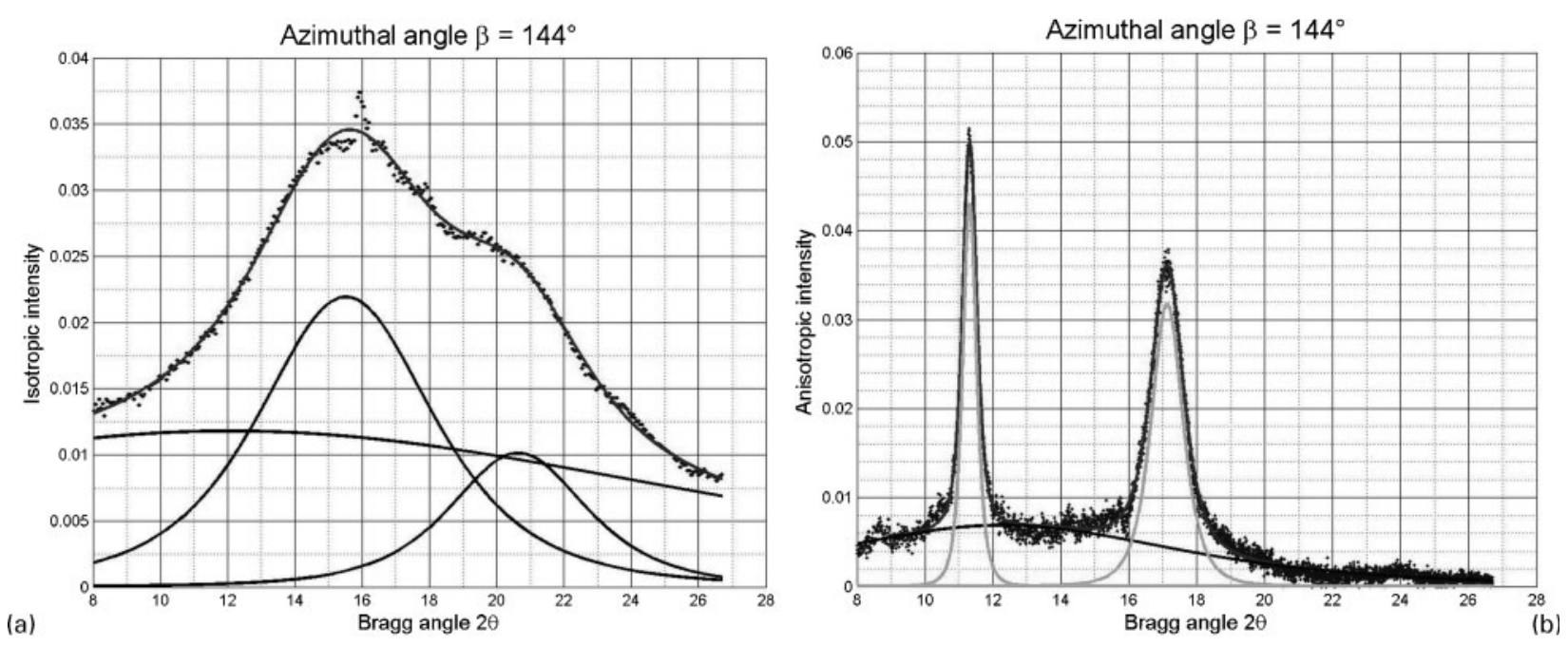

3 Examples of deconvolution of a spectrum $\left(2 \theta, l_{\text {isotropic }}\right)$ and $b$ spectrum $\left(2 \theta, l_{\text {anisotropic }}\right)$ fitted by series of Pearson functions

\section{Results}

The evolution of the index of crystallinity of carbon black filled NR during fatigue tests for different levels of deformation is given in Fig. 4. As expected, the larger the strain, the higher the crystallinity. During the three fatigue tests with the lowest minimum and maximum strain levels (test nos. 1, 2 and 3 ), the index of crystallinity decreases (of $15-50 \%$ ) during the first 250 cycles and then continues to decrease at a lower but constant rate until the end of the test. In Fig. 4, the results are shown only for the first 4000 cycles, but fatigue test no. 3 was performed during 40000 cycles, and the index of crystallinity decreases at a constant rate during the whole test, down to about $\chi=2 \%$ (in comparison with $\chi=7 \%$ at the beginning of the test). During fatigue test no. 4, which admits the highest minimum and maximum strain levels, the evolution of the index of crystallinity is very different: $\chi$ increases with the number of cycles. The minimum and maximum local stretch ratios reached during the fatigue tests have been measured by an optical technique, as well as the critical stretch ratios for crystallisation $\left(\lambda_{\mathrm{C}}\right)$ and melting $\left(\lambda_{\mathrm{M}}\right)$ at room temperature and low strain rates (measured with the same method as Trabelsi et al. ${ }^{8}$ ). Those stretch ratios are sketched in Fig. 5. It appears that the three fatigue tests for which the index of crystallinity decreases with the number of cycles have

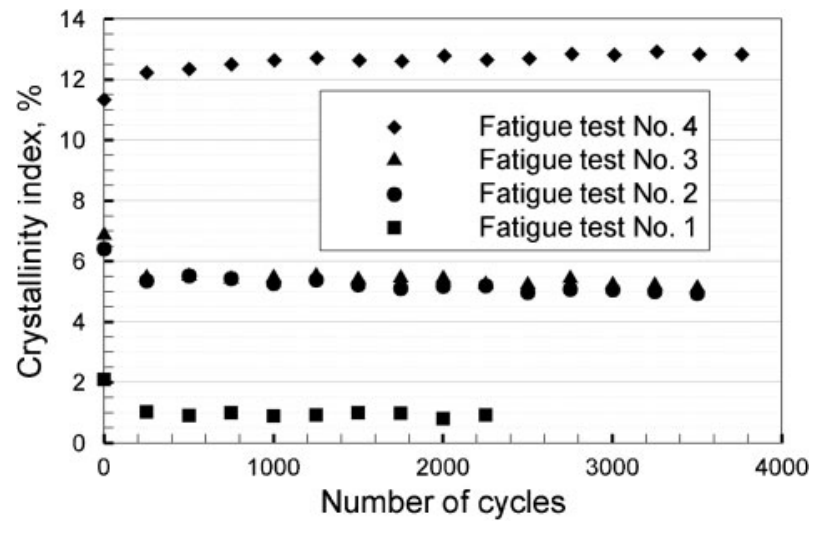

4 Evolution of crystallinity index during fatigue tests

been performed with minimum stretch ratios lower than $\lambda_{\mathrm{C}}$, whereas the minimum stretch ratio during test no. 4 , for which the index of crystallinity increases with the number of cycles, is greatly higher than $\lambda_{\mathrm{C}}$.

Figure $6 a-c$ shows the evolution of the mean size of the crystallites during fatigue test nos. 2, 3 and 4 respectively ( $\chi$ is too low during fatigue test no. 1 for accurate measurements of the crystallite size). Only the sizes in directions normal to the diffraction planes (200), (120) and (201) are shown because they correspond to the most intense diffraction arcs and hence to less scattered measurements. The crystallites size decreases with the number of cycles for fatigue test nos. 2 and 3 and increases for fatigue test no. 4. However, the sizes are very close for the three tests: $l_{200} \approx 125 \AA, l_{201} \approx 115 \AA$ and $l_{120} \approx 50 \AA$. Sizes in directions normal to the planes (121), (202) and (002), which correspond to less intense diffraction arcs, are not shown here but have been measured as well; they follow the same evolution $l_{200}$, $l_{201}$ and $l_{120}$, and their values are similar for the three tests as well.

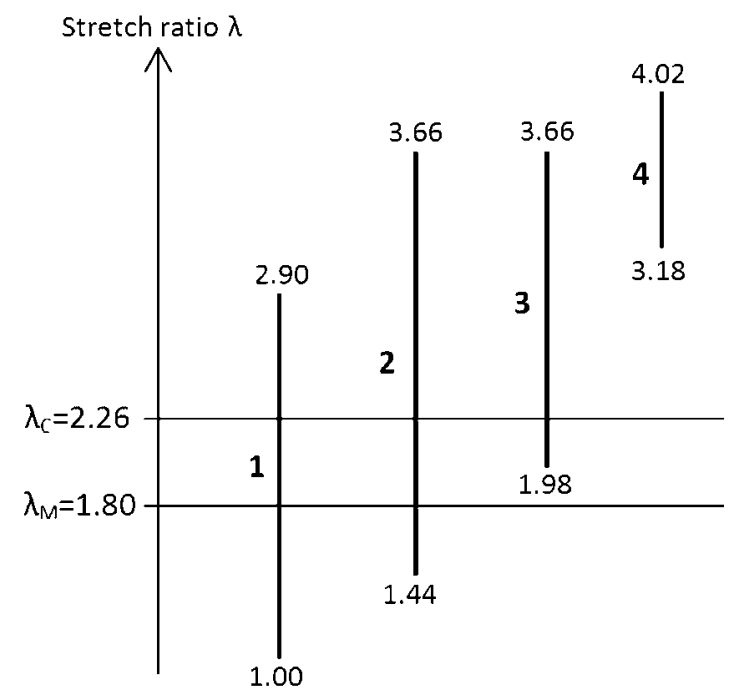

5 Minimum and maximum stretch ratios reached during fatigue tests compared with critical stretch ratios for crystallisation $\lambda_{c}$ and melting $\lambda_{M}$ (measured for quasistatic test): fatigue test numbers are in bold 

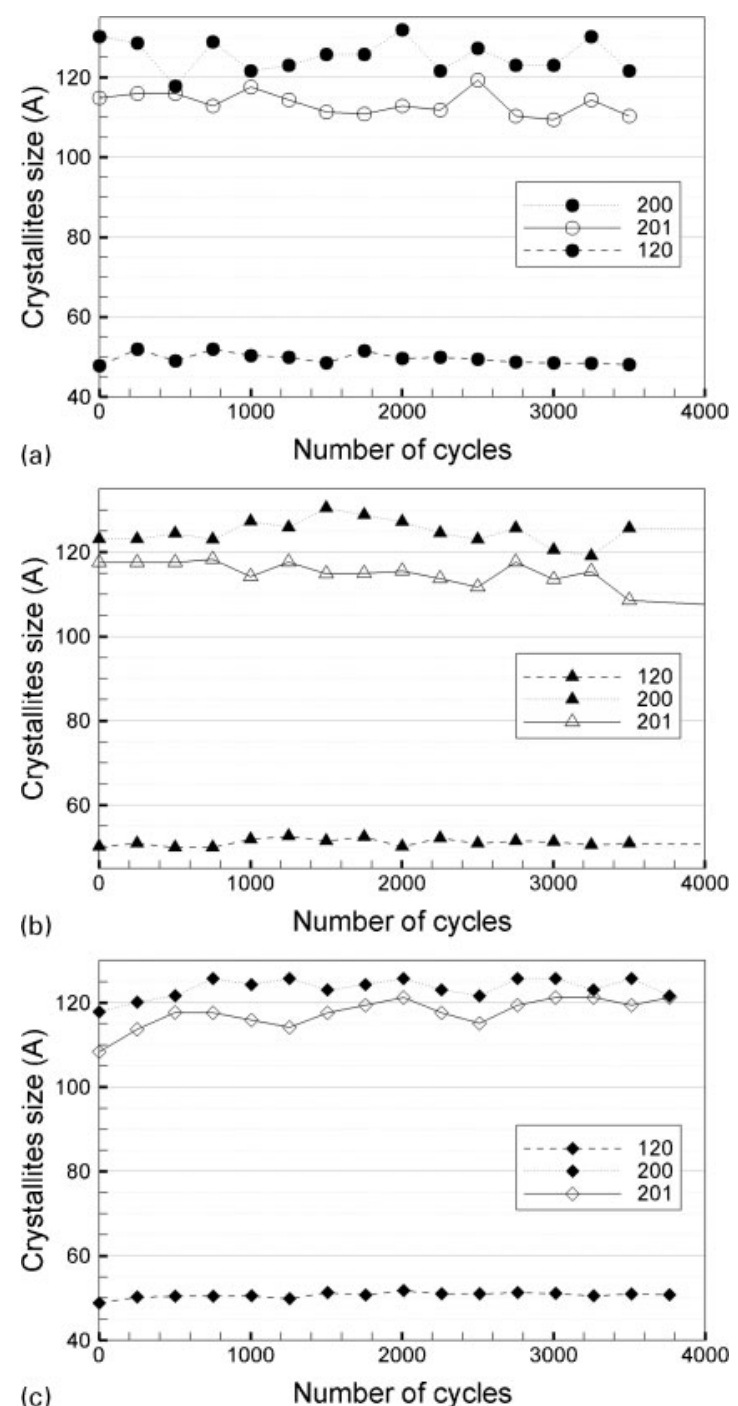

$a$ fatigue test no. 2; $b$ fatigue test no. $3 ; c$ fatigue test no. 4

6 Evolution of crystallite mean size during fatigue tests

The evolution of misorientation of the diffraction planes (200), (120) and (201) of the crystallites during fatigue test nos. 2, 3 and 4 is shown in Fig. 7. Misorientation of the crystallites from the mean orientation varies from \pm 9 to $\pm 11 \cdot 5^{\circ}$, depending on the diffraction planes and the fatigue tests considered. Nevertheless, for all fatigue tests and the three planes studied, and despite scattering of data, the same evolution is observed: the misorientation of the crystallites decreases with the number of cycles, particularly during the first 250 cycles of the fatigue tests.

\section{Discussion}

\section{Evolution of $\chi$ during fatigue tests}

Studying the fatigue of elastomers, experimenters and mechanicians face a recurring issue: it is extremely difficult to perform fatigue tests controlling any local mechanical quantity (such as stress, strain or energy). Indeed, fatigue tests are almost always performed controlling either the displacement of the machine grips or the force applied to the sample, from which local mechanical quantities can partially be calculated $a$ posteriori. In our study, fatigue tests were performed

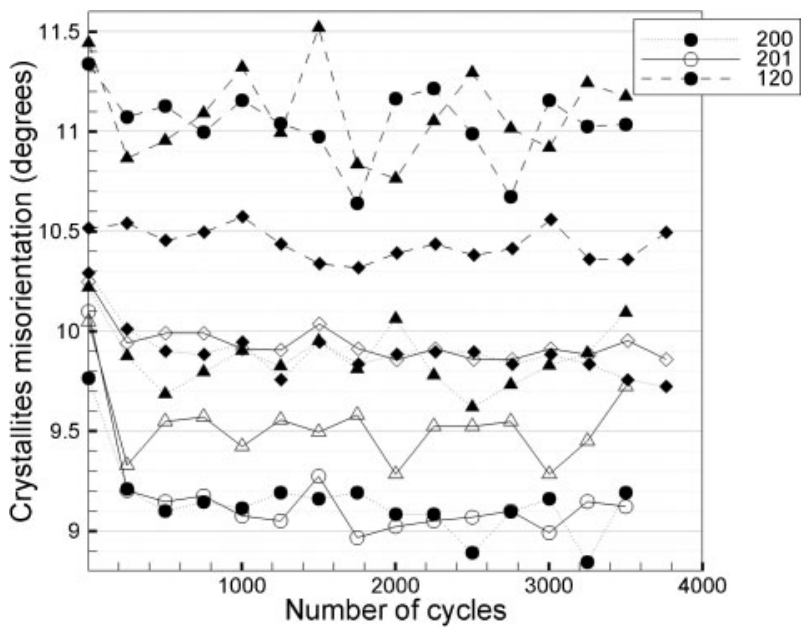

7 Evolution of crystallite misorientation during fatigue tests

by prescribing constant maximum and minimum displacements of the grips. As the material is cycled, stress softening and viscous effect modify both stress and strain reached at a given displacement for different numbers of cycles. This phenomenon can be minimised by precycling the material (to avoid the Mullins effect at the beginning of the test), but it cannot be completely suppressed. Thus, the evolution of the crystallinity index and the size and misorientation of crystallites during fatigue tests presented in the previous section were measured at constant displacement but at decreasing local stress and strain.

It is not yet established whether crystallisation of rubber is induced by strain, stress, energy or a combination of them. However, we believe that a mechanical quantity that controls crystallisation, even if undefined yet, exists; in the following, it is referred to as the 'crystallisation driving quantity'. As stress, strain and energy are closely related, we can assume that this crystallisation driving quantity evolves similarly as stress and strain during fatigue tests. As a consequence, the maximum value of this quantity reached at each cycle decreases too with the number of cycles during the fatigue tests. In the following, we discuss our results in the light of this assumption.

(i) As shown in Fig. 5, fatigue test nos. 1 and 2 are performed with minimum stretch ratios lower than the critical stretch ratio for melting $\lambda_{\mathrm{M}}$. Moreover, even if the initial minimum stretch ratio in fatigue test no. 3 is slightly higher than $\lambda_{\mathrm{M}}$, it becomes lower than $\lambda_{\mathrm{M}}$ after a few cycles. For these three tests, the same phenomenon takes place during each cycle: all the crystallites melt when the minimum displacement is reached, and new crystallites nucleate when the sample is stretched again. Nevertheless, as the maximum value of the crystallisation driving quantity reached during one cycle is lower than at the previous cycle, the crystallinity index is lower too. Hence, $\chi$ decreases with the number of cycles.

(ii) During fatigue test no. 4, local stress and strain decrease with the number of cycles as well, but the local stretch ratio is always greatly larger than $\lambda_{\mathrm{C}}$. In other words, the minimum value of the crystallisation driving quantity decreases from 


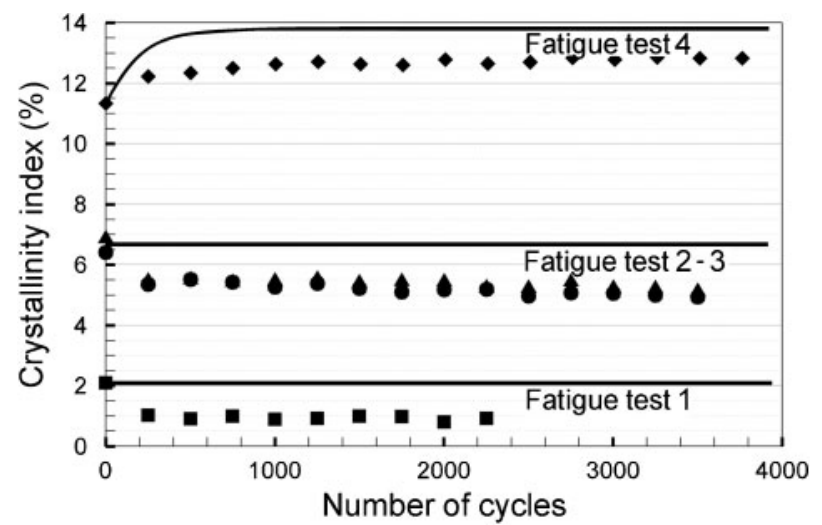

8 Evolution of crystallinity index with number of cycles during fatigue tests under constant crystallisation driving quantity as assumed by authors (lines) compared with original data measured during tests under constant displacement of grips (symbols)

one cycle to the next one, but it always remains higher than its threshold of crystallisation (the counterpart of $\lambda_{\mathrm{C}}$ ). As a consequence, when the grips are at minimum displacement, all crystallites do not melt (as $\lambda_{\mathrm{M}}$ is lower than $\lambda_{\mathrm{C}}$, the local stretch ratio is maintained higher than $\lambda_{\mathrm{M}}$ too). As the sample is stretched, the crystallisation driving quantity increases, as well as $\chi$, until the maximum displacement of the grips is reached. Then, when the sample is unstretched again, the crystallisation driving quantity decreases, and the change in $\chi$ results from the balance of two phenomena: on the one hand, $\chi$ decreases because the crystallisation driving quantity decreases, and on the other hand, the crystallisation driving quantity remains higher than the threshold of crystallisation, so crystallisation is still induced and $\chi$ continues to increase. Quantitatively, the latter phenomenon is not as significant as the first one, so $\chi$ decreases during the unstretching phase. However, this decrease during the unstretching phase is lower than the increase during the stretching phase. Hence, from one cycle to another, $\chi$ increases for the same value of crystallisation driving quantity. During fatigue test no. 4, this evolution of $\chi$ is minimised by the fact that the maximum crystallisation driving quantity reached during each cycle decreases during the test.

As stated previously, it would be very difficult to perform fatigue tests controlling the crystallisation driving quantity even if it was known. Nevertheless, in order to clarify our theory of crystallisation in fatigue, Fig. 8 shows our prediction of the evolution of $\chi$ with the number of cycles if the tests were performed at constant maximum and minimum values of crystallisation driving quantity (as compared to the original data measured during tests performed at constant maximum and minimum displacements of the grips). To summarise, four different situations are possible:

(i) The maximum crystallisation driving quantity is lower than the threshold of crystallisation: $\chi$ remains equal to 0 .

(ii) The maximum crystallisation driving quantity is higher than the threshold of crystallisation, and the minimum crystallisation driving quantity is lower than the threshold of melting (equivalent to test nos. 1, 2 and 3): all the crystallites melt at each cycle, and the maximum crystallinity index reached at each cycle is constant.

(iii) The maximum crystallisation driving quantity is higher than the threshold of crystallisation, and the minimum crystallisation driving quantity is between the thresholds of melting and crystallisation (this is not equivalent to test no. 3 , as during this test the minimum stretch ratio becomes lower than $\lambda_{\mathrm{M}}$ after a few cycles only): all the crystallites do not melt at each cycle, and crystallisation is induced during the unstretching phase of the cycles only at the beginning of the phase. It is not possible to conclude whether $\chi$ at maximum deformation is constant during the fatigue test or if it increases with the number of cycles.

(iv) Both minimum and maximum crystallisation driving quantities are higher than the thresholds of melting and crystallisation (equivalent to test No. 4): all the crystallites do not melt at each cycle, and crystallisation is induced during the unstretching phase. The maximum crystallinity index reached at each cycle increases with the number of cycles.

\section{Evolution of crystallite size and misorientation during fatigue tests}

We observe that the evolution of $\chi$ with the number of cycles is similar to the evolution of the size of the crystallites: they decrease when the minimum stretch ratio reached during the fatigue test is lower than $\lambda_{\mathrm{M}}$ and increase when it is higher than $\lambda_{\mathrm{C}}$. It suggests that the evolution of $\chi$ with the number of cycles is only due to a variation of the size of the crystallites, i.e. the number of macromolecules per crystallites, and not to the number of crystallites nucleating in the material at each cycle. Moreover, the misorientation of the crystallites has a similar evolution during the two types of fatigue test, but its value varies from one test to another, as shown in Fig. 7. We note that no correlation between the stretch ratios reached during fatigue and the level of misorientation is observed. An additional quasistatic test performed on the same material and in the same conditions shows that crystallites have very similar size and orientation during quasi-static tests as during fatigue tests. It suggests that once the crystallites are nucleated, cycling the material has little effect on the crystallite size and orientation.

\section{Conclusions}

In conclusion, we observe that during a fatigue test, the evolution of the index of crystallinity of NR with the number of cycles depends on the relative value of the minimum stretch ratio $\lambda_{\text {min }}$ compared with the threshold of melting $\lambda_{\mathrm{M}}$.

1. If $\lambda_{\min }<\lambda_{\mathrm{M}}$, then all the crystallites in the material melt at each cycle and then recrystallise, but are smaller and smaller at each cycle. It results in a decreasing index of crystallinity with the number of cycles.

2 . If $\lambda_{\min }>\lambda_{\mathrm{M}}$, then some crystallites never melt, even when the sample is stretched at $\lambda_{\text {min }}$. In this case, the mean size of the crystallites and then the index of crystallinity increase with the number of cycles. 
In addition, for both cases, the misorientation of the crystallites slightly decreases with the number of cycles: the $c$ axis of the crystallites is in average more and more parallel to the tension direction of the sample.

\section{References}

1. S. M. Cadwell, R. A. Merril, C. M. Sloman and F. L. Yost: Ind. Eng. Chem. Anal. Ed, 1940, 12, 19-23.
2. W. V. Mars and A. Fatemi: Rubber Chem. Technol., 2004, 77, 391. 3. H. Kawai: Rheol. Acta, 1975, 14, 27-47.

4. J. Y. Rouvière, A. Bennani, D. Pachoutinsky, J. Besson and S. Cantournet: Proc. Eur. Conf. on 'Constitutive models for rubber V', (ed. A. Boukamel et al.), 323-326; 2007, London, Taylor \& Francis.

5. S. Ran, D. Fang, X. Zong, B. S. Hsiao, B. Chu and P. F. Cunniff: Polymer, 2001, 42, (4), 1601-1612.

6. R. R. Reeber: J. Appl. Phys., 1970, 41, (13), 5063-5066.

7. J.-M. Chenal, C. Gauthier, L. Chazeau, L. Guy and Y. Bomal: Polymer, 2007, 48, (23), 6893-6901.

8. S. Trabelsi, P. A. Albouy and J. Rault: Macromolecules, 2003, 36, (20), 7624-7639.

9. J. Rault, J. Marchal, P. Judeinstein and P. A. Albouy: Eur. Phys. J. E, 2006, 21E, 243-261.

10. S. Toki, T. Fujimaki and M. Okuyama: Polymer, 2000, 41, 5423 5429.

11. G. R. Mitchell: Polymer, 1984, 25, 1562-1572.

12. A. Guinier: 'X-ray diffraction'; 1963 , New York, W. H. Freeman \& Co. 